\title{
Errata notice
}

Thomas J. Espenshade \& Charles A. Calhoun, An analysis of public opinion toward undocumented immigration, Population Research and Policy Review 12: 189-224 (1993).

The figures and appendix tables in this paper contain printer's errors. Corrected versions may be obtained from:

Thomas J. Espenshade, Office of Population Research, Princeton University, 21 Prospect Avenue, Princeton, NJ 08544-2091, USA.

Fax: (609) 258 1039; e-mail address: tje@opr.princeton.edu

The Publishers sincerely apologize for these mistakes. 